\title{
Efeitos de anisotropia sísmica VTI em perfis de impedância elástica
}

José Sampaio de Oliveira \& Jorge Leonardo Martins, Coordenação da Área de Geofísica, Observatório Nacional, MCT

Copyright 2010, SBGf - Sociedade Brasileira de Geofísica. Este texto foi preparado para a apresentação no IV Simpósio Brasileiro de Geofísica, Brasília, 14 a 17 de novembro de 2010. Seu conteúdo foi revisado pelo Comitê Técnico do IV SimBGf, mas não necessariamente representa a opinião da SBGf ou de seus associados. É proibida a reprodução total ou parcial deste material para propósitos comerciais sem prévia autorização da SBGf.

\section{RESUMO}

O entendimento dos mecanismos que induzem anisotropia sísmica é de fundamental importância na caracterização das formações sedimentares, principalmente se tais formações são acumuladoras de petróleo e gás. Neste trabalho, avaliamos os efeitos da anisotropia VTI em perfis impedância elástica. Usamos perfis geofísicos de poços perfurados em sedimentos turbidíticos na Bacia de Campos para estimar os parâmetros elásticos efetivos. A ocorrência de estratificação subhorizontal de camadas delgadas sustenta a consideração de simetria VTI na periferia dos poços investigados. A comparação ds perfis de impedância elástica isotrópica e anisotrópica resultantes evidenciam uma suave anisotropia VTI na formação estudada.

\section{INTRODUÇÃO}

Para a geofísica de exploração, a determinação dos mecanismos que induzem anisotropia sísmica em ambientes sedimentares sempre foi um desafio. Durante a aquisição de dados sísmicos, uma direção preferencial para propagação das ondas sônicas pode admitir como diagnóstico o alinhamento de fraturas nas formações geológicas. Portanto, a caracterização da direção preferencial do fraturamento é de primordial relevância para a implementação de métodos especiais de recuperação, pois o fluxo de fluidos tende a ocorrer ao longo de tais estruturações. No contexto do processamento dos dados sísmicos, desconsiderar a anisotropia sísmica conduz a erros no mapeamento de horizontes refletores, mascarando a geologia de subsuperfície. Quanto maior a intensidade da anisotropia, maiores serão os erros no mapeamento dos horizontes refletores na secção sísmica final.

$\mathrm{Na}$ literatura, podemos encontrar diversos trabalhos que enfocam o estudo de anisotropia sísmica. O trabalho de Thonsen (1986), por exemplo, é considerado um marco no estudo dos parâmetros que controlam a simetria anisotrópica mais comum em ambientes sedimentares, a simetria VTI (vertical transverse isotropy). Dessa forma, a partir do trabalho de Thomsen, o processamento sís- mico sofreu um revolução pois permitiu introduzir parâmetros de anisotropia VTI para fins de incorporar efeitos anisotrópicos no mapeamento dos horizontes refletores.

Nesse trabalho, usamos o conceito de impedância elástica isotrópica, derivada por Connolly (1998) e posteriormente adaptada por Martins (2006) para meios anisotrópicos, para construir perfis na periferia de poços. As tendências de variação de anisotropia em um ambiente sedimentar podem ser investigadas diretamente por meio de perfis de impedância elástica anisotrópica. A formulação desta propriedade física depende das magnitudes das densidades e das velocidades de propagação de ondas $\mathrm{P}$ e S. A intensidade da anisotropia é pode ser observada nos perfis de impedância elástica. A intercalação de diferentes litotipos em profundidade no ambiente sedimentar também é um mecanismo indutor de anisotropia sísmica. Camadas rochosas originadas por diferentes processos de estratificação normalmente possuem valores distintos para os parâmetros que caracterizam a impedância elástica (isto é: densidade e velocidades sísmicas), tornando possível a interpretação dessas estruturas.

Para um melhor desenvolvimento e aprimoração de técnicas que visem a otimização do imageamento da subsuperfície, é essencial o conhecimento das direções preferenciais de anisotropia. Essa avaliação pode ser realizada satisfatoriamente por meio de perfis de impedância elástica conforme apresentado nas seções seguintes.

\section{METODOLOGIA}

A fim de construir perfis de impedância elástica contendo efeitos de anisotropia sísmica VTI, usamos como dados de entrada perfis geofísicos de densidade efetiva $\rho$, e de velocidades compressionais $\alpha$ e cisalhantes $\beta$. Seguimos a metodologia proposta em Liner e Fei (2006), isto é: (1) Aplicação da média de Backus (Postma, 1955; Backus, 1962), para estimativa de parâmetros elásticos efetivos para a simetria anisotrópica VTI. (2) Aplicação da fórmula da impedância elástica anisotrópica especificada para meios VTI.

A equação da impedância elástica isotrópica foi apresentada inicialmente por Connolly (1998), definindo-a como um atributo petrofísico com propriedades semelhantes a da impedância acústica. Consistentemente, a impedância acústica se reduz à impedância elástica para incidências normais. O resultado da formulação de Connolly mostra que a impedância elástica isotrópica depende dos valores da densidade efetiva da rocha, das velocidades de propagação das ondas primárias e secundárias e do ângulo de incidência $\theta$. A forma modificada da equação da impedância elástica isotrópica é 
expressa por (Whitcombe, 2002; Martins, 2003):

$$
E I(\theta)=E I_{0}\left(\frac{\rho \alpha}{\rho_{0} \alpha_{0}}\right)\left(\frac{\alpha}{\alpha_{0}}\right)^{\tan ^{2} \theta}\left(\frac{\mathrm{G}}{\mathrm{G}_{0}}\right)^{-\xi} .
$$

O termo $\mathrm{G}$ representa o módulo de cisalhamento, $\mathrm{G}=$ $\rho \beta^{2}$. O parâmetro $\xi$ também é função do ângulo de incidência $\theta$, ou seja, $\xi \equiv \xi(\theta)=4 k_{1} \sin ^{2} \theta$. A constante $k_{1}$ é definida utilizando a média das velocidades $\alpha$ e $\beta$ : $k_{1}=(\bar{\beta} / \bar{\alpha})^{2}$.

Martins (2006) apresenta uma equação para a impedância elástica que incorpora efeitos de anisotropia sísmica para qualquer que seja a simetria anisotrópica. Escrevendo em uma forma condensada, a equação da impedância elástica anisotrópica é dada por:

$$
E I(\phi, \theta)=E I(\theta) \Delta E I(\phi, \theta),
$$

onde o termo $E I(\theta)$ representa a impedância isotrópica conforme expressa na equação (1). O termo adicional $\Delta E I(\phi, \theta)$, que denota a correção devido aos efeitos anisotrópicos, além da dependência do ângulo de incidência $\theta$ também traz informação da direção de investigação (azimute) $\phi$. A correção $\Delta E I(\phi, \theta)$ é expressa em Martins (2006) da seguinte forma:

$$
\Delta E I(\phi, \theta)=\exp \left[2 \int d R_{P P}(\phi, \theta)\right] .
$$

Para qualquer que seja a simetria anisotrópica, o argumento da função exponencial é expresso por

$$
\begin{aligned}
& 2 \int d R_{P P}(\phi, \theta) \equiv \alpha^{2} k_{2}\left\{\epsilon_{z} \cos ^{2} \theta\right. \\
& +\left[\left(\delta_{x}-8(\beta / \alpha)^{2} \gamma_{x}\right) \cos ^{2} \phi\right. \\
& +\left(\delta_{y}-8(\beta / \alpha)^{2} \gamma_{y}\right) \sin ^{2} \phi \\
& \left.+\left(\chi_{z}-4(\beta / \alpha)^{2} \epsilon_{45}\right) \sin 2 \phi\right] \sin ^{2} \theta \\
& +\left[\epsilon_{x} \cos ^{4} \phi+\epsilon_{y} \sin ^{4} \phi+(1 / 4) \delta_{z} \sin ^{2} 2 \phi\right. \\
& \left.\left.+\left(\epsilon_{16} \cos ^{2} \phi+\epsilon_{26} \sin ^{2} \phi\right) \sin 2 \phi\right] \sin ^{2} \theta \tan ^{2} \theta\right\} .(4)
\end{aligned}
$$

Adicionalmente, $k_{2}=1 / \bar{\alpha}^{2}$, e os termos $\epsilon_{x}, \epsilon_{y}, \epsilon_{z}, \epsilon_{16}$, $\epsilon_{26}, \epsilon_{45}, \gamma_{x}, \gamma_{y}, \delta_{x}, \delta_{y}, \delta_{z}$ e $\chi_{z}$ são definidos como parâmetros de anisotropia fraca (Pšenčík and Gajewski, 1998):

$$
\begin{gathered}
\epsilon_{x}=\frac{A_{11}-\alpha^{2}}{2 \alpha^{2}}, \epsilon_{y}=\frac{A_{22}-\alpha^{2}}{2 \alpha^{2}}, \epsilon_{z}=\frac{A_{33}-\alpha^{2}}{2 \alpha^{2}}, \\
\delta_{x}=\frac{A_{13}+2 A_{55}-\alpha^{2}}{\alpha^{2}}, \delta_{y}=\frac{A_{23}+2 A_{44}-\alpha^{2}}{\alpha^{2}}, \\
\delta_{z}=\frac{A_{12}+2 A_{66}-\alpha^{2}}{\alpha^{2}}, \\
\chi_{z}=\frac{A_{36}+2 A_{45}}{\alpha^{2}}, \epsilon_{16}=\frac{A_{16}}{\alpha^{2}}, \epsilon_{26}=\frac{A_{26}}{\alpha^{2}}, \\
\gamma_{x}=\frac{A_{55}-\beta^{2}}{2 \beta^{2}}, \gamma_{y}=\frac{A_{44}-\beta^{2}}{2 \beta^{2}} \text { e } \epsilon_{45}=\frac{A_{45}}{\beta^{2}} .
\end{gathered}
$$

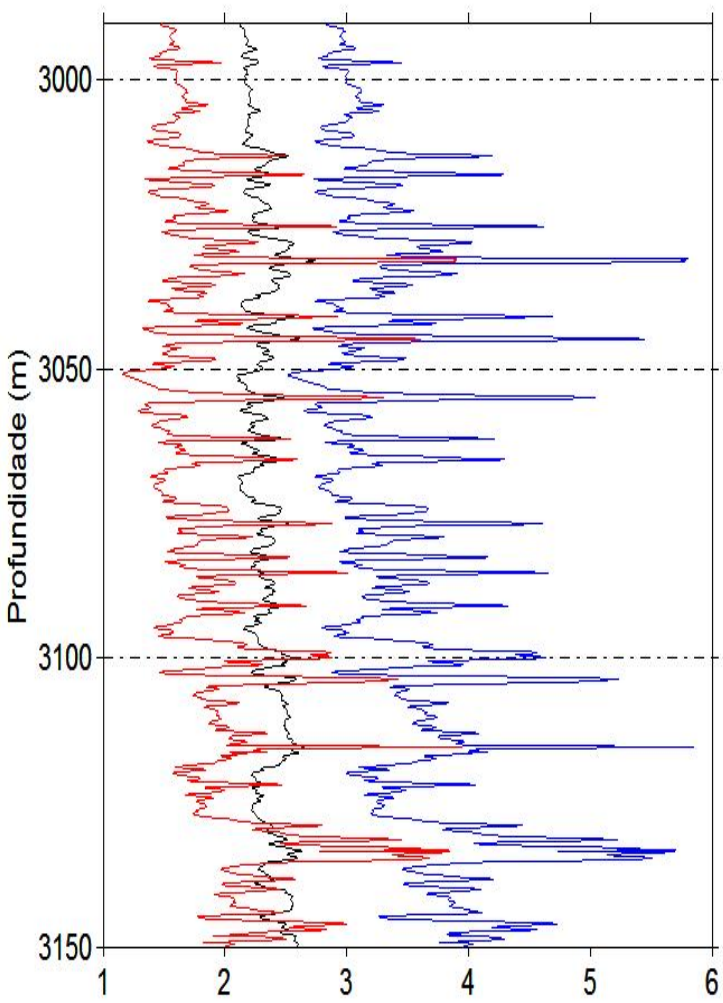

Figura 1: Perfis geofísicos extraídos do poço NA 01 do Campo de Namorado, Bacia de Campos. Em azul e vermelho perfis de ondas primárias e secundárias, respectivamente, em $\mathrm{km} / \mathrm{s}$. Em preto perfil de densidades em $\mathrm{g} / \mathrm{cm}^{3}$.

Assim, a informação da anisotropia do meio é trazida para a impedância elástica pelos parâmetros de anisotropia fraca, através dos parâmetros elásticos normalizados pela densidade $A_{i, j}$ (em notação consensada). Nessas circunstâncias, os termos $\alpha$ e $\beta$ passam a ser velocidades de referência, cuja escolha é feita pelo intérprete.

Dessa forma, para especificação da equação (4) para simetria VTI, a matriz elástica fica definida como: $A_{11}=$ $A_{22}, A_{13}=A_{23}, A_{33}, A_{44}=A_{55}, A_{66}$ e $A_{12}=A_{11}-$ $2 A_{66}$. Consequentemente, $\chi_{z}=\epsilon_{16}=\epsilon_{26}=\epsilon_{45}=0$, $\epsilon_{y}=\epsilon_{x}, \delta_{y}=\delta_{x}, \delta_{z}=2 \epsilon_{x}$ e $\gamma_{y}=\gamma_{x}$ são os parâmetros de anisotropia fraca que controlam a equação da impedância elástica anisotrópica. A expressão da correção $\Delta E I(\phi, \theta)^{\mathrm{vti}}$ será

$$
\begin{aligned}
& 2 \int d R_{P P}(\theta) \equiv \alpha^{2} k_{2}\left[\epsilon_{z} \cos ^{2} \theta\right. \\
& \left.+\left(\delta_{x}-8(\beta / \alpha)^{2} \gamma_{x}\right) \sin ^{2} \theta+\epsilon_{x} \sin ^{2} \theta \tan ^{2} \theta\right] .(10)
\end{aligned}
$$

Observamos na equação (10) que a dependência da direção de investigação $\phi$ deixa de existir, conforme o pressuposto para simetria VTI, isto é, meio azimutalmente isotrópico. Além disso, apenas os parâmetros 

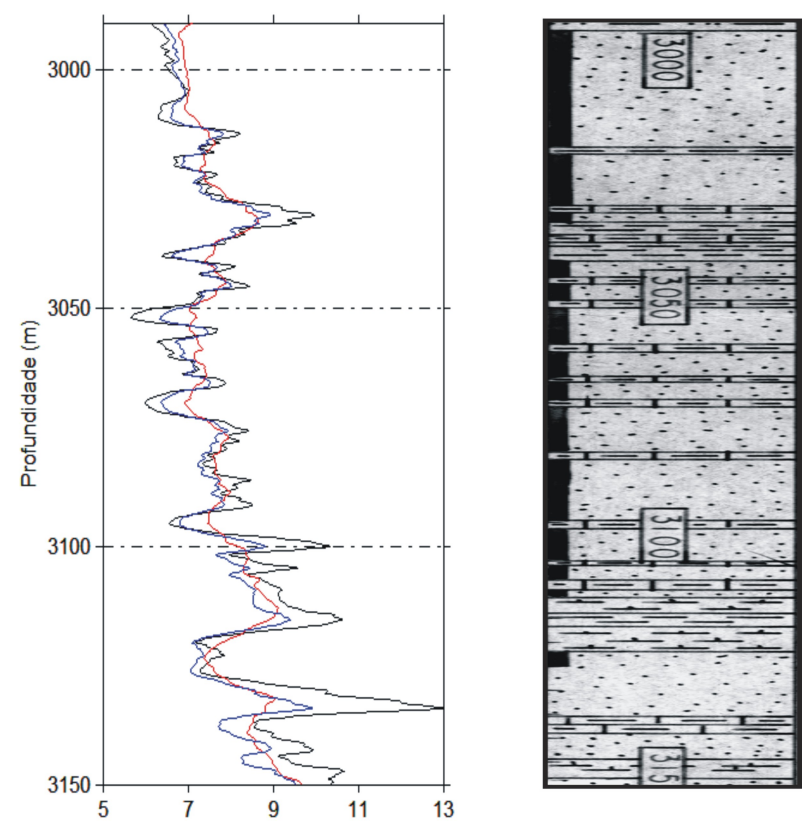

Figura 2: Perfis de impedância elástica (em $\mathrm{km} / \mathrm{s} \times$ $\mathrm{g} / \mathrm{cm}^{3}$ ), e perfil composto para o poço NA 01 do Campo de Namorado, Bacia de Campos. Em preto e vermelho perfis de impedância elástica anisotrópica considerando ângulos de incidência de $\theta=0^{\circ}$ e $\theta=30^{\circ}$ respectivamente. Em azul perfil de impedância elástica isotrópica com ângulo de incidência $\theta=30^{\circ}$.

de anisotropia fraca $\epsilon_{z}, \delta_{x}, \gamma_{x}$ e $\epsilon_{x}$ interferem no cálculo da impedância elástica em meios VTI. No que se refere às velocidades de referência, assumimos, após alguns experimentos, $\alpha^{2}=\left(A_{11}+A_{22}+A_{33}\right) / 3$ e $\beta^{2}=$ $\left(A_{44}+A_{55}+A_{66}\right) / 3$.

\section{RESULTADOS}

Usando a metodologia descrita em Liner e Fei (2006), construímos perfis de impedância elástica isotrópica e anisotrópica a partir de perfis de densidades e velocidades primárias e secundárias de 2 poços no reservatório Namorado, bacia de Campos. O campo de petróleo fica localizado a $80 \mathrm{~km}$ da costa brasileira, na localidade do município de Macaé. Os poços utilizados para construção dos perfis foram o NA 01, localizado pela coordenada UTM $(354.575,751.737) \mathrm{km}$, e NA 02 localizado pela coordenada $(352.964,751.732) \mathrm{km}$. A figura 1 mostra os perfis correspondentes ao poço NA 01 . Nas figuras 2 e 3 , podemos notar as facies na periferia de ambos os poços (perfis compostos). A estratigrafia é tipicamente subhorizontal, composta por estratificação de camadas delgadas. Esse tipo de estruturação geológica induz anisotrópia sísmica com eixo de simetria vertical, ou simplesmente simetria VTI (Postma, 1955; Liner and Fei, 2006). Os tipos litológicos dessa bacia sedimentar são na maioria formados por rochas areníticas geradas predominantemente por correntes de tur-
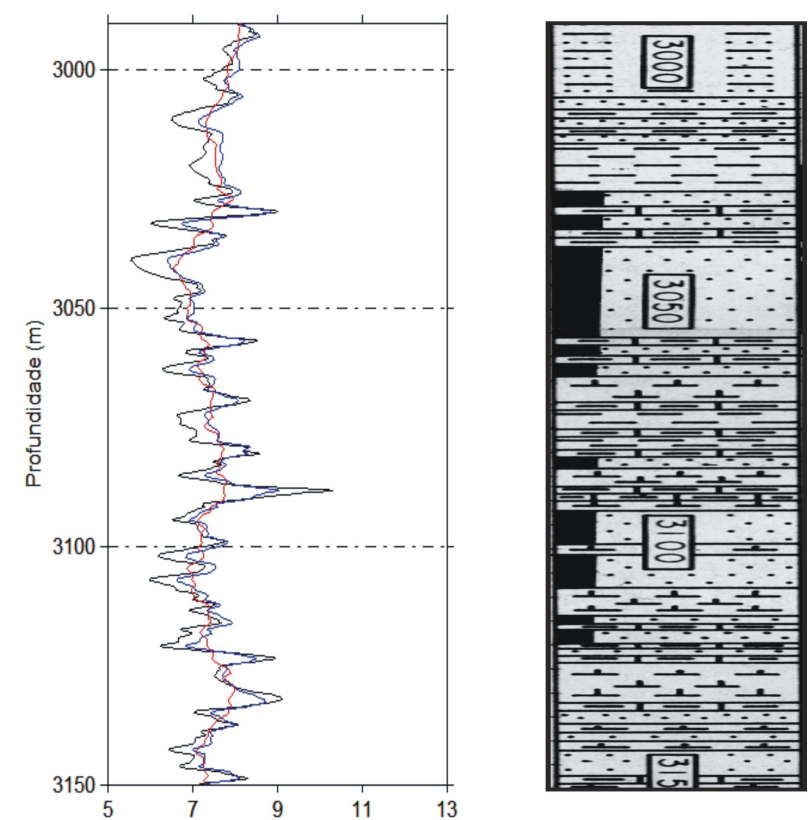

Figura 3: Perfis de impedância elástica estimados em $\mathrm{km} / \mathrm{s} \times \mathrm{g} / \mathrm{cm}^{3}$, e perfil composto para o poço NA 02 do Campo de Namorado, Bacia de Campos. Em preto e vermelho perfis de impedância elástica anisotrópica considerando ângulos de incidência de $\theta=0^{\circ}$ e $\theta=30^{\circ}$ respectivamente. Em azul perfil de impedância elástica isotrópica com ângulo de incidência $\theta=30^{\circ}$.

bidez. O intervalo sedimentar onde se insere o reservatório fica de $2940 \mathrm{~m}$ à $3300 \mathrm{~m}$ de profundidade na formação Macaé superior e sua área com potencial para exploração abrange em torno de $24 \mathrm{~km}^{2}$.

A estimativa dos perfis de impedância elástica isotrópica e anisotrópica foram realizadas em um intervalo de $160 \mathrm{~m}$ dos poços a partir da profundidade de $2990 \mathrm{~m}$, correspondente a um trecho atravessado pelo reservatório. As medidas realizadas para construção dos perfis de densidades e de velocidades de ondas primárias foram efetuadas a cada $0.15 \mathrm{~m}$ de profundidade, totalizando 1051. O perfil de velocidade de ondas secundárias inicialmente era inexistente, e sua estimativa foi realizada conforme Lee (2006). À esquerda das figuras 2 (poço NA 01) e 3 (poço NA 02), mostramos os perfis de impedância elástica isotrópica e anisotrópica para os ângulos de incidência de $0^{\circ}$ e $30^{\circ}$. Os perfis de impedância construídos utilizando ângulo de incidência normal, fazem a investigação da propriedade física nas imediações do ponto da aquisição dos dados, enquanto que os perfis construídos utilizando o ângulo de incidência de $30^{\circ}$ são interpretados como sendo a avaliação da impedância em pontos mais afastados.

Alguns dos valores de maior relevância do tratamento estatístico dado a esses perfis podem ser observado nas tabelas (1) e (2). Pode-se verificar que nos dois poços 
utilizados, os valores máximo e mínimo de impedância elástica isotrópica e anisotrópica a $30^{\circ}$ são muito próximos entre si assim como seus valores médios. Essa resposta do ambiente sedimentar para a modelagem anisotrópica da impedância elástica depende dos valores assumidos pelos parâmetros de anisotropia, conforme é discutido nas discussões e conclusões.

Tabela 1: Medidas estatísticas para perfis de impedância elástica do poço NA 01. Valores de impedância medidos em $\mathrm{km} / \mathrm{s} \times \mathrm{g} / \mathrm{cm}^{3}$.

\begin{tabular}{ccc}
\hline $\begin{array}{c}\text { Medidas } \\
\text { estatísticas }\end{array}$ & $E I^{i s o}\left(30^{\circ}\right)$ & $E I^{v t i}\left(30^{\circ}\right)$ \\
\hline \hline$E I_{\min }$ & 6.33 & 6.78 \\
$E I_{\max }$ & 9.91 & 9.66 \\
$E I_{\operatorname{med}}$ & 7.68 & 7.89 \\
$E I_{\text {var }}$ & 0.55 & 0.41 \\
$E I_{\sigma}$ & 0.74 & 0.64 \\
\hline
\end{tabular}

Tabela 2: Medidas estatísticas para perfis de impedância elástica do poço NA 02. Valores de impedância medidos em $\mathrm{g} / \mathrm{cm}^{3} \times \mathrm{km} / \mathrm{s}$.

\begin{tabular}{ccc}
\hline $\begin{array}{c}\text { Medidas } \\
\text { estatísticas }\end{array}$ & $E I^{\text {iso }}\left(30^{\circ}\right)$ & $E I^{v t i}\left(30^{\circ}\right)$ \\
\hline \hline$E I_{\min }$ & 6.42 & 6.56 \\
$E I_{\max }$ & 9.04 & 8.55 \\
$E I_{\text {med }}$ & 7.72 & 7.55 \\
$E I_{\text {var }}$ & 0.26 & 0.17 \\
$E I_{\sigma}$ & 0.51 & 0.41 \\
\hline
\end{tabular}

\section{DISCUSSÃO E CONCLUSÕES}

A análise conjunta dos perfis de impedância elástica isotrópica e anisotrópica permite ao interpretador a determinação de intervalos com maior indução de anisotropia sísmica VTI. Pode-se notar nos perfis compostos, que os intervalos constituídos por lâminas delgadas (na maioria folhelhos), se correlacionam com intervalos nos perfis de impedância cujos valores isotrópico e anisotrópico para impedância de mesma incidência possuem uma variação entre sí. Essa diferença pode ser interpretada como sendo originada pelo nível de indução anisotrópica nos perfis de impedância elástica. Essa informação é trazida pelos parâmetros de anisotropia utilizados. Caso seus valores tendessem a zero não haveria diferença entre os perfis de impedância. Utilizando o mesmo raciocínio, pode-se concluir que os intervalos correspondentes a rochas areníticas, de acordo com os perfis compostos, possuem baixa indução de anisotropia sísmica VTI, visto que os valores absolutos de impedância isotrópica e anisotrópica são muito próximos. De forma geral, é possível concluir que os poços avaliados são constituídos por intervalos predominantemente isotrópicos, cons- tituídos por arenítos, intercalados com intervalos predominantemente anisotrópicos, onde encontram-se tipos delgados de rochas.

Pode-se verificar também, que no topo de camadas muito espessas ocorrem variações bruscas nos valores de impedância. Essa propriedade dos perfis de impedância pode servir como parâmetro adicional na estimativa da delimitação de camadas tratadas pela sísmica tradicional. Poder-se-á em um estudo mais aprofundado, determinar o tipo litológico a partir da diferença absoluta entre os perfis isotrópicos e anisotrópicos, utilizando como princípio de estudo a indução anisotrópica originada pelo alinhamento de minerais em simetrias genéricas.

\section{AGRADECIMENTOS}

Neste trabalho, apresentamos alguns dos resultados do projeto de pesquisa "Caracterização de Anisotropia Sísmica Utilizando Perfis Geofísicos de Poços de Petróleo e Gás", que obteve suporte financeiro do CNPq (proc. 471647/2006-3). José Sampaio de Oliveira agradece ao auxílio financeiro oferecido pela CAPES para o desenvolvimento de dissertação de mestrado Curso de PG em Geofísica no Observatório Nacional.

\section{REFERÊNCIAS}

Backus, G. E., 1962, Long-wave elastic anisotropy produced by horizontal layering: J. Geophys. Res., 67, 4427-4440.

Connolly, P., 1998, Calibration and inversion of non-zero offset seismic: 68th Ann. Internat. Mtg. SEG - Expanded Abstracts, 68th SEG Meeting: Expanded Abstracts, 182-184.

Lee, M., 2006, A simple method to predicting s-wave velocity:, 71, 161-164.

Liner, C. L., and Fei, T. W., 2006, Layer-induced seismic anisotropy from full-wave sonic logs: theory, application and validation:, 71, D183-D190.

Martins, J. L., 2003, A second-order approach for P-wave elastic impedance technology:, 47, 545-564.

Martins, J. L., 2006, Elastic impedance in weakly anisotropy media:, 71, D73-D83.

Postma, G. W., 1955, Wave propagation in a stratified medium:, 20, 780-806.

Pšenčík, I., and Gajewski, D., 1998, Polarization, phase velocity and NMO velocity of $\mathrm{qP}$ waves in arbitrary weakly anisotropic media:, 63, 1754-1766.

Thomsen, L., 1986, Weak elastic anisotropy:, 51, 19541966.

Whitcombe, D. N., 2002, Elastic impedance normalization:, 67, 60-62. 